\title{
Front end for D-band High Data Rate Point to Point links
}

\author{
Rupa Basu ${ }^{1}$, Jeevan N. Rao ${ }^{1}$, Rosa Letizia ${ }^{1}$, Qiang Ni ${ }^{2}$, Edward Wasige ${ }^{3}$, Abdullah Al-Khalidi ${ }^{3}$, \\ Jue Wang ${ }^{3}$, Claudio Paoloni ${ }^{1}$ \\ ${ }^{1}$ Engineering Department, Lancaster University, Lancaster, UK \\ ${ }^{2}$ School of Computing and Communications, Lancaster University, Lancaster, UK \\ ${ }^{3}$ High Frequency Electronics Group, School of Engineering, University of Glasgow, Glasgow, UK
}

\begin{abstract}
A novel front end for high data rate point to point links at D-band (151 - $174.8 \mathrm{GHz})$ will provide up to $45 \mathrm{~Gb} / \mathrm{s}$ over $1 \mathrm{~km}$ range. The low power signal generation is provided by a low cost Resonant Tunnelling Diode (RTD) oscillator. The RTD oscillator is connected to a Traveling Wave Tube that provides up to $10 \mathrm{~W}$ transmission power.
\end{abstract}

\section{INTRODUCTION}

$\mathrm{T}$ HE electronics for the exploitation of the spectrum above $100 \mathrm{GHz}$ for responding to the huge traffic growth that $5 \mathrm{G}$ and future $6 \mathrm{G}$ applications $[1,2]$ will bring is still at laboratory level $[3,4]$. One of the main obstacles to the deployment of millimeter wave wireless links is the high path loss and rain attenuation and the lack of transmission power at Watt level. This prevents the availability of high signal to noise ratio (SNR) needed to enable high order modulation schemes and high spectral efficiency. The signal generation is a further challenge above $100 \mathrm{GHz}$. New and simple modulation approaches for multi Gigabit per second $(\mathrm{Gb} / \mathrm{s})$ data rate are needed for a wide adoption of millimeter wave links.

This paper describes a novel D-band wireless system in point to point, to provide $45 \mathrm{~Gb} / \mathrm{s}$ data rate over 1 kilometer link, to offer an affordable alternative to fiber, both to solve deployment issues and for flexible and affordable installation [5].

Frequency Division Duplex is used over two D-band wide frequency bands, $151.5-161.5 \mathrm{GHz}$ and $161.5-174.8 \mathrm{GHz}$. Each frequency band is served by a front end including a transmitter in one band and a receiver for the other frequency band. In the following a short description of the system, its components and the main results will be presented.

\section{D-BAND WIRELESS LINK}

The DLINK wireless systems uses two different front ends with the same topology, but different frequency structure. Each front end consists of two main complementary components, one at low power and one at high power. One is a D-band Resonant Tunneling Diode (RTD) transmitter $[6,7]$ with integrated a vector modulator at $\mathrm{XQAM}$ level (with $\mathrm{x}$ could be 16 or 64). This circuit permits to simplify the transmitter topology, removing mixer and amplifier, with enhancement of the low phase noise oscillator specifications for high order modulation schemes.

The second enabling component is a traveling wave tube (TWT) that provides the required level of transmission power to satisfy the link budget in real condition with $99.99 \%$ availability in ITU zone up to K (rain attenuation at $140 \mathrm{GHz}$ about $17 \mathrm{~dB}$ ).

A traveling wave tube is a vacuum electronics device able to generate output power higher than one or two orders of

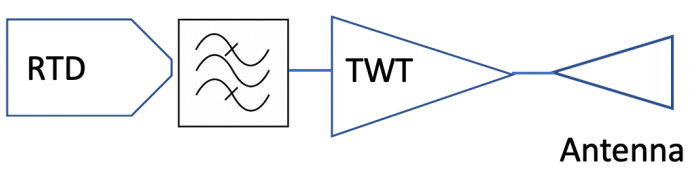

TX LB $151.5-161.5 \mathrm{GHz}$

TX UB $161.5-174.8 \mathrm{GHz}$

Fig. 1. Front end schematic: TX LB: Transmitter Lower Band, TX UB: Transmitter Upper Band

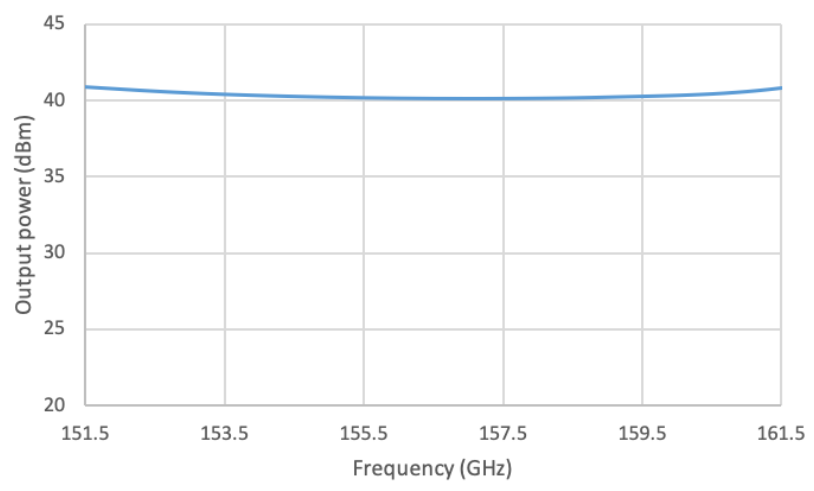

Fig. 2. Output power lower band TWT

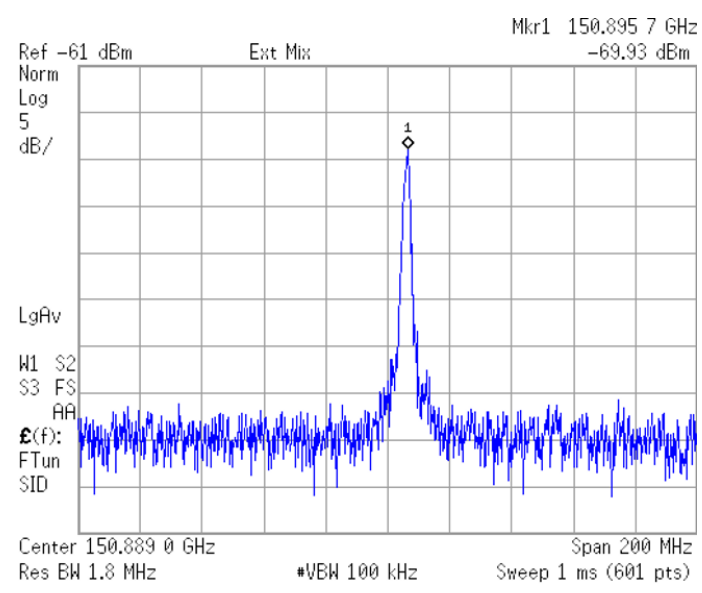

Fig. 3. Measured spectrum of D-band RTD transmitter 
magnitude of solid state amplifiers at the same frequency. Only a few prototypes were reported at D-band [8]. Two different TWTs are designed to cover the two frequency bands (151.5 $161.5 \mathrm{GHz}$ and $161.5-174.8 \mathrm{GHz}$ ) with minimum $10 \mathrm{~W}$ saturated in their respective operating frequency band. A horn antenna with 38 - 40dBi gain ensures high directivity but easy alignment.

All the parts of the D-band wireless system have been designed and are in fabrication phase.

\section{RESULTS}

The TWT was designed by using the double corrugated waveguide [9]. Fig.2 shows the output power of the lower band TWT (151.5 - 161.5 GHz) higher than 40dBm (10W) over the full band. A D-band RTD oscillator has been designed and characterized on wafer. The design details can be found in $[6$, 7]. The total device size is about $500 \times 600 \mathrm{um}^{2}$. The measured spectrum is shown in Fig. 3. The oscillator will be used in the front end to drive TWT.

\section{CONCLusions}

The first D-band link in point to point based on a RTD oscillator amplified by a traveling wave tube has been presented. The system is in development phase. A field trial is planned for a test in real environment.

\section{ACKNOWLEDGMENT}

The DLINK project is funded by EPSRC grants EP/S009620/1 and $\mathrm{EP} / \mathrm{S} 009442 / 1$.

\section{REFERENCES}

[1] Cisco Visual Networking Index: Global Mobile Data Traffic Forecast Update, 2016-2021 White Paper, 2017

[2] Dang, S., Amin, O., Shihada, B. et al. What should 6G be?. Nat Electron 3, 20-29 (2020). https://doi.org/10.1038/s41928-019-0355-6

[3] Nagatsuma, Tadao, Guillaume Ducournau, and Cyril C. Renaud. "Advances in terahertz communications accelerated by photonics." Nature Photonics 10, no. 6, pp. 371-379, 2016.

[4] Dhillon, S. S., et al. "The 2017 terahertz science and technology roadmap." Journal of Physics D: Applied Physics, 50.4, pp. 043001, 2017).

[5] Claudio Paoloni, Rupa Basu, Laxma Reddy Billa, Jeevan Mahadev Rao, Rosa Letizia, Qiang Ni, Edward Wasige, Abdullah Al-Khalidi and Jue Wang' "Long range millimeter wave wireless links enabled by traveling wave tubes and resonant tunnelling diodes", UCMMT 2019, London, August 2019.

[6] J. Wang, A. Al-Khalidi, L. Wang, R. Morariu, A. Ofiare and E. Wasige, "15-Gb/s 50-cm Wireless Link Using a High-Power Compact III-V 84GHz Transmitter," in IEEE Transactions on Microwave Theory and Techniques, vol. 66, no. 11, pp. 4698-4705, Nov. 2018.

[7] A. Al-Khalidi et al., "Resonant Tunneling Diode Terahertz Sources With up to $1 \mathrm{~mW}$ Output Power in the J-Band," in IEEE Trans. on Terahertz Science and Technology, vol. 10, no. 2, pp. 150-157, March 2020

[8] Z. Wang et al., "Development of a 140-GHz folded-waveguide traveling wave tube in a relatively larger circular electron beam tunnel," $J$. Electromagn. Waves Appl., vol. 31, no. 17, pp. 1914-1923, 2017.

[9] M. Mineo and C. Paoloni, "Double Corrugation Rectangular Waveguide Slow-wave Structure for THz Vacuum Devices", IEEE Trans. on Electron Devices, pp.3169-3175, November 2010. 